\title{
The Research on the De-Noising Method for Signals Based On CEEMD
}

\author{
Wei Huang ${ }^{1, a,{ }^{*}}$, Wei-chang $\mathrm{Xu}^{1, \mathrm{~b}}, \mathrm{Ya}$-ke $\mathrm{Wu}^{1, \mathrm{c}}$, Hong-li Hao ${ }^{1, \mathrm{~d}}$ \\ ${ }^{1}$ High-tech Institute, Fan Gong-ting South Street on the 12th, Qing Zhou, Shan Dong, China \\ a172026399@qq.com, bwuyake8211@163.com, 'mm678xu@163.com, d282408122@qq.com
}

Keywords: vibration signal; de-noising; CEEMD; random noise

\begin{abstract}
Dynamical properties of mechanical systems can be obtained with the vibration signals from the systems. However, for the influence of noises, it is difficult to accurately acquire the features. Therefore, de-noising operation is significant for vibration signal in the practical engineering. In order to resolve this problem, the Complementary Ensemble Empirical Mode Decomposition (CEEMD) method is introduced to try to eliminate noises from the analyzed signal. At first, the theory of the method is illustrated, which included adding white noises, EMD for the signal and calculating the mean of the intrinsic mode function. On this base, the signal which contains several harmonic components with white noise is processed by CEEMD. As the result shown, the random noise can be effectively removed; moreover, the harmonic components can be accurately separated. And these improve that the CEEMD is an effective method for the de-noising.
\end{abstract}

\section{Introduction}

Vibration signals are important data for the analysis to the dynamic features of the mechanical systems. But, the signal will be polluted by random noises in the collection process, which can result in reducing the analysis precision and accuracy. At present, de-nosing processing is a key point of signal processing, and the scholars, at home and abroad, were working in this, such as discrete wavelet transform [1], stationary wavelet transform [2], wavelet packet [3] and Winner filtering [4] and other de-noising methods. The properties of signals were not taken into consideration in these methods; therefore, the universality is not good. In the end of 20th century, Empirical Mode Decomposition (EMD) was proposed by Huang [4], based on the Hilbert transform, in which, intrinsic mode function is the decomposition unit. This method is the general expression form for the decomposition operation based on the inner product. As driven by the data itself, the universality of algorithm is excellent. However, for the influence of the modal aliasing, the application range is limited. The property that all frequencies are contained in white noise is masterly utilized by $\mathrm{Wu}$, and the Ensemble Empirical Mode Decomposition was proposed in 2008 [5]. The method can reduce modal aliasing, which expand the application scope of the EMD, and improve the de-noising effect. Yeh, etc. further improve the EEMD. And the algorithm can remove the surplus noise introduced by the adding white noise, and the computation time is less, which is called (Complementary Ensemble Empirical Mode Decomposition, CEEMD). In this paper, the theory of the algorithm is illustrated and the de-noising effect was demonstrated.

\section{The Principle Of The CEEMD}

Aim to overcome the harm of the modal aliasing, a data-analysis algorithm assisted by noises is proposed, and called CEEMD. The property that white noise contains equally distributed energy in all frequencies is made used in the CEEMD. The specific processing step is as followed: (1)Add low-energy white noises to the original in plus and minus; (2)Employ the EMD to the signal added with noise, and a group of IMFs are obtained (the specific steps of the EMD can be found in literature [4]); (3)Repeat the step1 and step 2, and add different random noise series to the original signal, and there operations are repeated $\mathrm{N}$ times; (4)Add the IMF with same number together that obtained in $2 \mathrm{~N}$-times EMD decomposition, and the means of the IMFs are calculated, and are taken as the final IMF: 


$$
C_{j}(t)=\frac{1}{N} \sum_{i=1}^{N} C_{i j}(t)
$$

Where, $C_{\mathrm{j}}(t)$ represents the ith IMF in the CEEMD decomposition.

\section{The Simulate Signal}

In order to verify the effectiveness of the CEEMD, the simulate signal is made, and the formula is as followed

$$
x=\sin (10 \cdot 2 \pi t)+\sin (20 \cdot 2 \pi t)+5 \sin (40 \cdot 2 \pi t)
$$

The white noise with certain degree energy is added to the signal. There sampling time is $8 \mathrm{~s}$, and the sample period is 0.004s. The time series figure of the signal is shown in Fig.1.

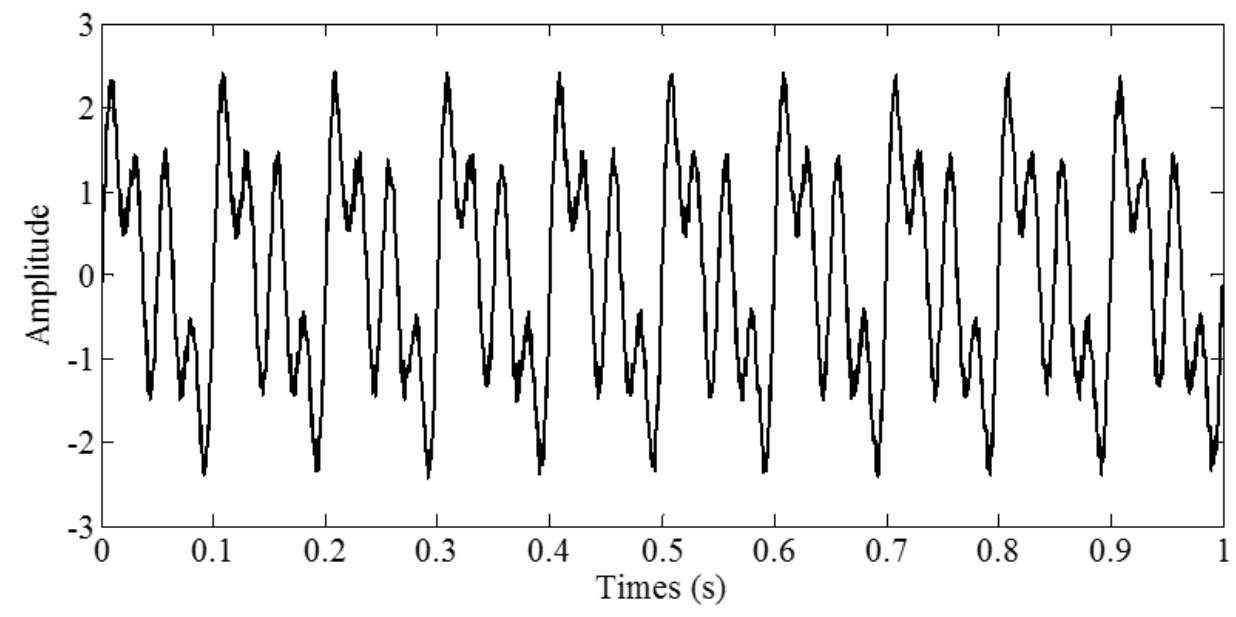

Fig.1 The time series figure of $\mathrm{x}$ sign with white noises

\section{CEEMD Decomposition}

The CEEMD is applied to the signal, and the amplitude of the white noise that is added to the signal is $30 \%$ of the standard deviation of the processed signal, and the EMD decomposition is conducted for 100 times. The data is expended by mirror method to reduce the affect of the boundary effect. 12 IMFs are obtained in the decomposition. As revealed in Ref. [6], an IMF with a high coefficient of correlation between it and the analyzed signal means that this IMF is an important component. Fig. 2 presents the coefficients of correlation between different IMFs and the original signal. We can learn that IMFs 6-8 are vital components of the signal, as the coefficients of correlation are relatively greater. So these IMFs are discussed. The valuable IMFs are shown in Fig.2. As shown in Fig.3, after CEEMD decomposition operation, the noise is separated, and the harmonic component at 10,20 and $40 \mathrm{~Hz}$ are also separated.

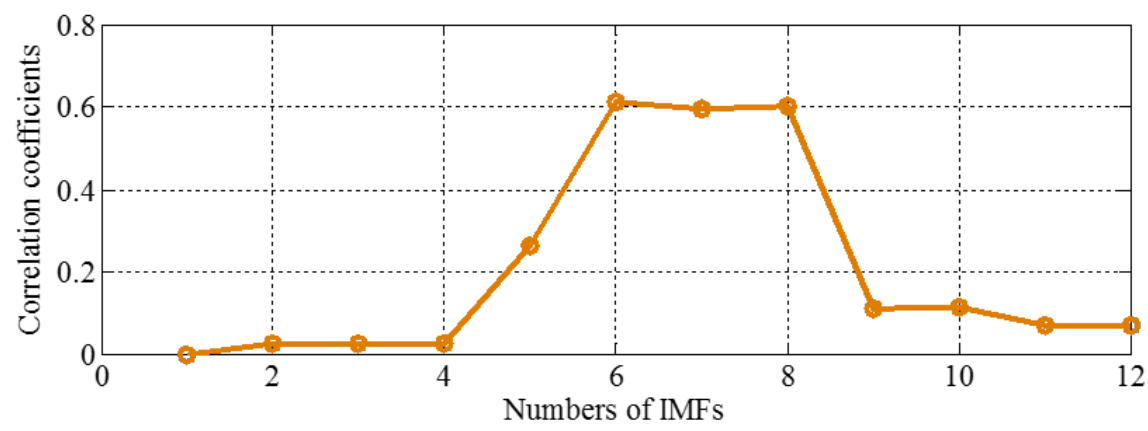

Fig.2 Correlation coefficients between each IMF and the original signal 

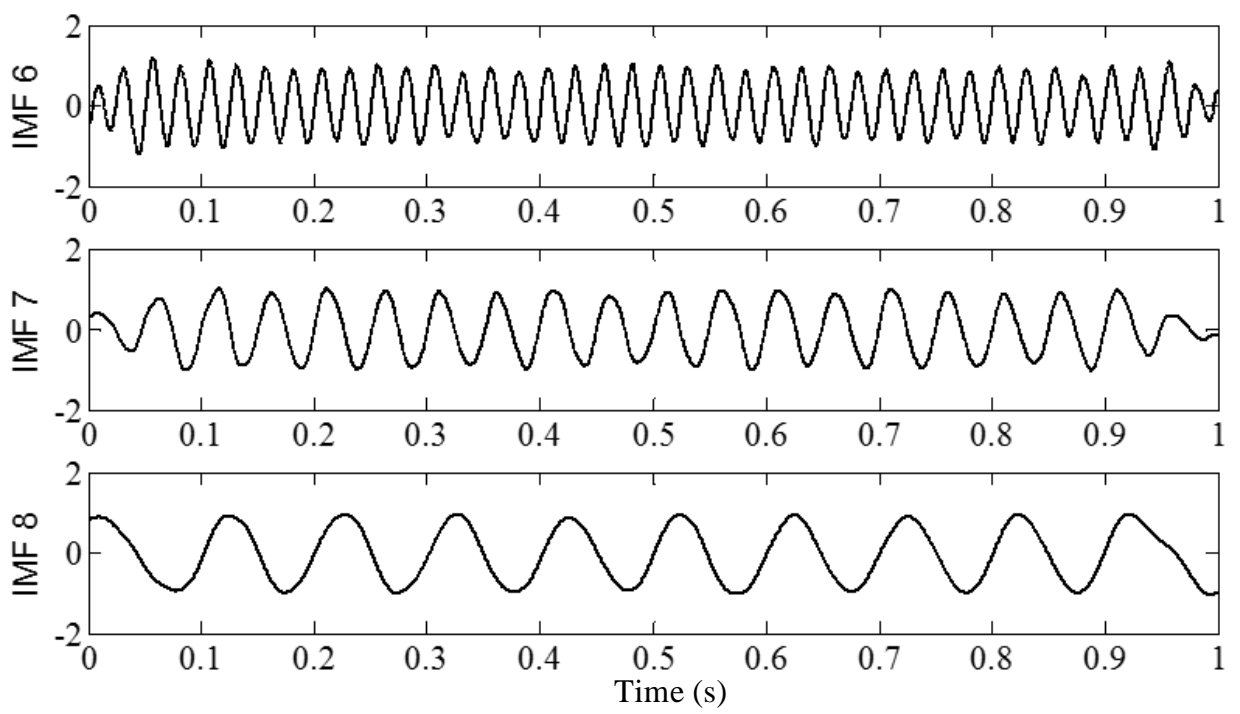

Fig.3 The figure of the valuable IMFs

\section{The Contrast between the Processed Signal and the Original Signal}

As shown in Fig.2, it is easy to learn that IMF 1-5 and IMF 9-12 are the noise components. Add the remnant IMF together, and the de-noising signal is obtained, and it is shown in Fig.2. Comparing Fig.4 and Fig.1, it can be known that the signal become smooth, after de-noising operation.

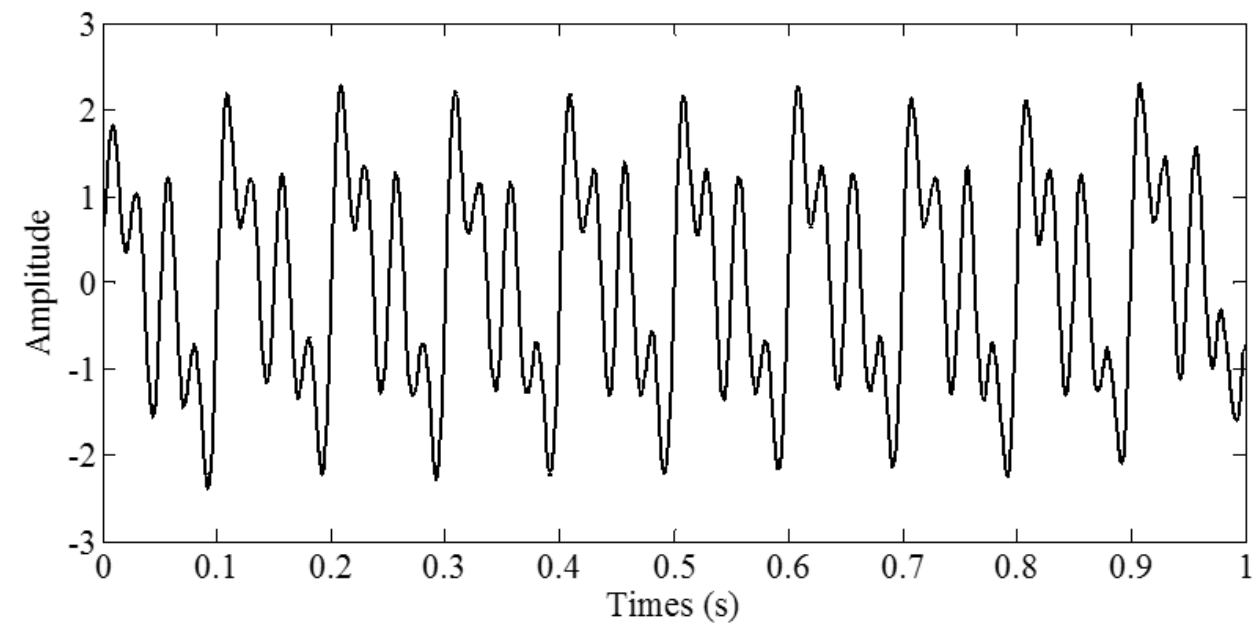

Fig.4 The de-noising figure

\section{Summary}

In order to eliminate noise from the vibration signal, the CEEMD is introduced in this paper. At first, the principle of the algorithm is illustrated, and the specific decomposition process is elaborated. The method is applied to a simulate signal, which contain harmonic components and white noise. It can be concluded that the result shows that the noise can be effectively eliminated by using this method. However, as shown in Fig.3, a decomposition error that part of harmonic is separated can be found in the right endpoint, which results from the endpoint effect. And this problem should be further studied.

\section{References}

[1] Chun-xiang Shi,Qi-feng Luo. Hilbert-huang transform and wavelet analysis of time history signal[J]. Acta Seismologica Sinica . 2003 (4). 
[2] XIA Chen-xi, YANG Jun, LI Shun-bo, Application of Best Wavelet Packet Basis Algorithm in De-noising of Blasting Vibration Signal[J]. Blasting.2011(3):4-7.

[3] Liu Tao, Zhang Wei, Yan Shaoze. A novel image enhancement algorithm based on stationary wavelet transform for infrared thermography to the de-bonding defect in solid rocket motors[J]. Mechanical Systems and Signal Processing, 2015, 62: 366-380.

[3] ZHANG Feng;MA Shu-xiao;SHI Xian-feng. Algorithm of Vibration Signal Based on Cricular Wiener Filtering[J].2014(24).6:49-55.

[4] Huang, N. E., Z. Shen, S. R. Long, M. C. Wu, E. H. Shih, Q. Zheng, C. C. Tung, and H. H. Liu, 1998: The empirical mode decomposition and the Hilbert spectrum for nonlinear and nonstationary time series analysis. Proceedings of the Royal Society A, London, 454, 903-995.

[5] Liu Tao, Yan Shaoze, Zhang Wei. Time-frequency analysis of nonstationary vibration signals for deployable structures by using the constant-Q nonstationary gabor transform[J]. Mechanical Systems and Signal Processing, 2016, 75: 228-244.

[6] Wu, Z., and N. E. Huang, 2009: Ensemble Empirical Mode Decomposition: a noise-assisted data analysis method. Advances in Adaptive Data Analysis, 1, 1-41.

[7] Torres M E, Colominas M A, Schlotthauer G, et al. A complete ensemble empirical mode decomposition with adaptive noise[C]//2011 IEEE International Conference on Acoustics, Speech and Signal Processing (ICASSP). IEEE, 2011: 4144-4147. 